\title{
Mechanical Response of Porous Scaffolds for Cartilage Engineering
}

\author{
J. JANČÁŔ ${ }^{1}$, A. SLOVÍKOVÁ ${ }^{1}$, E. AMLER ${ }^{2}$, P. KRUPA ${ }^{3}$, H. KECOVÁ ${ }^{4}$, \\ L. PLÁNKA ${ }^{5}$, P. GÁL $^{5}, A$. NEČAS ${ }^{4}$
}

${ }^{1}$ Institute of Materials Chemistry, University of Technology, Brno, ${ }^{2}$ Department of Biophysics, Second Medical Faculty, Charles University, Prague, ${ }^{3}$ Department of Medical Imaging and Radiology, St. Anne's University Hospital, Masaryk University, Brno, ${ }^{4}$ Department of Surgery and Orthopedics, Small Animal Clinic, Faculty of Veterinary Medicine, University of Veterinary and Pharmaceutical Sciences Brno, ${ }^{5}$ Department of Pediatric Surgery, Orthopedics and Traumatology, Masaryk University, Brno, Czech Republic

Received May 23, 2007

Accepted May 29, 2007

On-line available May 31, 2007

\begin{abstract}
Summary
Mechanical properties of scaffolds seeded with mesenchymal stem cells used for cartilage repair seem to be one of the critical factors in possible joint resurfacing. In this paper, the effect of adding hyaluronic acid, hydroxyapatite nanoparticles or chitosan nanofibers into the cross-linked collagen I on the mechanical response of the lyophilized porous scaffold has been investigated in the dry state at $37{ }^{\circ} \mathrm{C}$ under tensile loading. Statistical significance of the results was evaluated using ANOVA analysis. The results showed that the addition of hyaluronic acid significantly $(p<<0.05)$ reduced the tensile elastic modulus and enhanced the strength and deformation to failure of the modified cross-linked collagen I under the used test conditions. On the other hand, addition of hydroxyapatite nanoparticles and chitosan nanofibers, respectively, increased the elastic modulus of the modified collagen ten-fold and four-fold, respectively. Hydroxyapatite caused significant reduction in the ultimate deformation at break while chitosan nanofibers enhanced the ultimate deformation under tensile loading substantially $(p<<0.05)$. The ultimate tensile deformation was significantly $(p<<0.05)$ increased by addition of the chitosan nanofibers. The enhanced elastic modulus of the scaffold was translated into enhanced resistance of the porous scaffolds against mechanical load compared to scaffolds based on cross-linked neat collagen or collagen with hyaluronic acid with similar porosity. It can be concluded that enhancing the rigidity of the compact scaffold material by adding rigid chitosan nanofibers can improve the resistance of the porous scaffolds against compressive loading, which can provide more structural protection to the seeded mesenchymal stem cells when the construct is implanted into a lesion. Moreover, scaffolds with chitosan nanofibers seemed to enhance cell growth compared to the neat collagen I when tested in vitro as well as the scaffold stability, extending its resorption to more than 10 weeks.
\end{abstract}

\section{Key words}

Chitosan $\bullet$ Nanofibers $\bullet$ Hyaluronic acid $\bullet$ Hydroxyapatite $\bullet$ Mesenchymal stem cells $\bullet$ Joint resurfacing 


\section{Introduction}

Damaged or diseased articular cartilage frequently leads to progressive joint debilitation resulting in a marked decrease in the quality of life. Tissue engineering, a budding field in modern biomedical sciences, promises creation of viable substitutes for failing organs or tissues. It represents the amalgamation of rapid developments in cellular and molecular biology, on the one hand, and material, chemical and mechanical engineering, on the other. Current tissue engineering approaches are mainly focused on the restoration of pathologically altered tissue structure based on the transplantation of cells in combination with supportive matrices and biomolecules. The ability to manipulate and reconstitute tissue structure and function in vitro has tremendous clinical implications and is likely to have a key role in cell and gene therapies in the coming years (Risbud and Sittinger 2002)

Articular cartilage is a hypocellular, hydrated connective tissue that provides a low-friction, wearresistant, load-bearing surface in synovial joints and exhibits characteristic mechanical properties. The thickness, density, and alignment of collagen fibrils also differ from the articular surface, where fibrils are oriented parallel to the articular surface, to the deep layer, where fibrils are perpendicular to the boundary between bone and cartilage. The relationship between the apparent properties of full-thickness articular cartilage and those of partial-thickness sections is unclear. The properties of the articular cartilage are very much dependent on its porous structure and the extremely hydrophilic proteoglycan content. Collagen and proteoglycnas form about $30 \%$ of the cartilage tissue. The remaining $70 \%$ is water and the ability of proteoglycans to immobilize water in the cartilage is the most important feature of the healthy cartilage (Martin et al. 1998). The mechanical properties of cartilage depend on the composition and microstructural organization of its material constituents. For example, elastic moduli under compression are attributed to the swollen proteoglycan/water gel trapped by the collagen network, while tensile moduli are thought to be due primarily to the collagen network. However, the details of this dependence are unclear. It is not known whether the total amount of a constituent or details of its organization is the relevant microstructural parameter. Compression tests have often been performed to assess the biomechanical properties of full-thickness articular cartilage. Full-thickness bovine articular cartilage was tested by oscillatory confined compression superimposed on a static offset up to $45 \%$, and the data fit to estimate the modulus, permeability, and electrokinetic coefficient, assuming homogeneity. Additional tests on partialthickness cartilage were then performed to assess the depth- and strain-dependent properties in an inhomogeneous model, assuming three discrete layers. Estimates of the zero-strain equilibrium-confined compression modulus, $H_{\mathrm{A} 0}$, differered among the individual layers of cartilage and full-thickness cartilage. $H_{\mathrm{A} 0}^{i}$ increased from $0.27 \mathrm{MPa}$ in the surface layer to 0.71 $\mathrm{MPa}$ in the subchondral area, and bracketed the apparent homogeneous value $(0.47 \mathrm{MPa})$. These results help to interpret the biomechanical assessment of full-thickness articular cartilage (Chen et al. 2001).

One has to consider the cartilage as a higly viscoelastic gel-like material with the water phase being held in the cartilage tissue by electrostatic interactions between proteoglycans and water molecules, resulting in a strong dependence of the viscoelastic moduli on the deformation rate. Hence, at slow strain rates encountered in standard mechanical tests, the tensile elastic modulus of the articular cartilage reaches only $0.5-1.6 \mathrm{MPa}$ (Mow et al. 1991). The aggregate compressive modulus of the articular cartilage is around $0.9 \mathrm{MPa}$ while at impact speeds simulating physiological loading rates, the compressive modulus can reach up to 100-500 $\mathrm{MPa}$ (Martin et al. 1998). Moreover, the values measured for cartilage tissue are "effective" values since the cartilage consists of several layers with different elastic moduli in different directions. Under tensile loading, it is the connective tissue, i.e., collagen fibers, that carries the load. Under compressive load, it is the ability of proteoglycans to restrict water mobility that affects the elastic modulus of the cartilage and tissue permeability becomes the main structural variable.

The tensile stiffness of cartilage tissue grown from chondrocyte culture was both measured experimentally and predicted using a composite-model theory, relating tissue microstructure to macroscopic material stiffness. The tissue was altered by several treatment protocols to provide a wide range of collagenfibril volume fractions $(0.015-0.15)$. The rate of change of tissue modulus with change in collagen volume fraction predicted by the theory was within $14 \%$ of the slope of the linear fit through the experimental data, without the use of fitting parameters for the theoretical value of the slope (Simha et al. 1999).

The depth dependence of material properties of articular cartilage, known as the zonal differences, is incorporated into a nonlinear fibril-reinforced poroelastic 
model developed previously in order to explore the significance of material heterogeneity in the mechanical behavior of cartilage. The material variations proposed are based on extensive observations. The collagen fibrils are modeled as a distinct constituent which reinforces the other two constituents representing proteoglycans and water. The Young modulus and Poisson's ratio of the drained nonfibrillar matrix are so determined that the aggregate compressive modulus for confined geometry fits the experimental data. Three nonlinear factors are considered, viz. the effect of finite deformation, the dependence of permeability on dilatation and the fibril stiffening with its tensile strain. Solutions are extracted using a finite element procedure to simulate unconfined compression tests. The features of the model are then demonstrated with emphasis on the results obtainable only with a nonhomogeneous model, showing reasonable agreement with experiments. The model suggests mechanical behavior significantly different from that displayed by homogeneous models: not only the depth variations of the strains which are expected by qualitative analyses but also, for instance, the relaxation-time dependence of the axial strain which is normally not expected in a relaxation test. Therefore, such a nonhomogeneous model is necessary for better understanding of the mechanical behavior of cartilage ( $\mathrm{Li}$ et al. 2000). Theoretically, the linear biphasic model of articular cartilage indicates that the instantaneous mechanical response of articular cartilage is equivalent to that of an incompressible elastic material (Toyras et al. 2001).

Specially designed biomaterial scaffolds are one of the key components in tissue engineering. Research is focused on developing bioresorbable scaffolds that exhibit optimal physical properties coupled with excellent biocompatibility. Scaffolds act as shape and guidance templates for in vitro and in vivo tissue development (Lee et al. 2003). For cartilage and bone tissues, a suitable scaffold provides initial mechanical stability and supports even cell distribution. Natural polymeric gels, such as hyaluronic acid, collagen, alginate (Dorotka et al. 2005) and chitosan, have been used successfully (Risbud and Sittinger 2001). These scaffolds permit 3D immobilization of cells and maintain the differentiated phenotype of chondrocytes (Chen et al. 2007). However, their mechanical behavior is insufficient for tissue transplantation and so solid bioresorbable fiber scaffolds or other porous structures are used to achieve initial biomechanical stability (Williams et al. 2004). Synthetic biodegradable poly $(\alpha$-hydroxy esters) such as polylactic acid (PLLA), polyglycolic acid (PGA) and copolymer PLGA (Courtney et al. 2006) have been used extensively in this context. Both types of material increase proteoglycan synthesis compared with collagen scaffolds (Fang et al. 2005). Injectable in situ cross-linkable polymeric preparations that entrap cells have been designed (Temeno et al. 2000) and techniques that combine the advantages of both porous fiber structures and gels are being explored as suitable alternatives to either gels or fiber scaffolds (Korhonen et al. 2002). Research is also focused on developing 'smart scaffolds' that incorporate inflammatory inhibitors or antibiotics. Slow and controlled release of these bioactive molecules provides sufficient time for the new cartilage to adapt and mature in a 'hostile' in vivo situation or to prevent early infection after surgery. The key to successful repair and regeneration of cartilage is to provide the repair site with sufficient chondrogenic cells in a suitable delivery vehicle to ensure maximum differentiation and deposition of right extracellular matrix. New optimized culture methodologies and bioreactors that provide appropriate mechanical and other guidance clues must be engineered to ensure the successful function of the engineered tissue. As we gain more and more information about the identity of all morphogens for chondrocyte differentiation it might be possible to orchestrate massive cartilage regeneration by clever combination of smart 3D scaffolds and such morphogenic factors. The management of cells both in situ and ex vivo, will be crucial to the success of such tissue engineering efforts (Risbud and Sittinger 2002).

Hybrid scaffolds composed of $\beta$-chitin and collagen were prepared by combining salt-leaching and freeze-drying methods. The chitin scaffold used as a framework was easily formed into desired shapes with a uniformly distributed and interconnected pore structure with average pore size of $260-330 \mu \mathrm{m}$. The mechanical strength and the rate of biodegradation increased with the porosity, which could be modulated by the salt concentration. In addition, atelocollagen solution was introduced into the macropores of the chitin scaffold to improve cell attachment. Web-like collagen fibers fabricated between pores of chitin were produced by a 0.1 $\%$ collagen solution, whereas a $0.5 \%$ collagen solution only coated the surface of the chitin scaffold. After 3 days of culture, fibroblasts cultured in collagen-coated scaffolds were attached at the place where the collagen was fabricated, whereas cells did not attach and aggregate on the scaffold of chitin alone. After 14 days, the fibroblasts showed a good affinity for and proliferation on all collagen-coated chitins (Lee et al. 2004). 
One of the main issues in tissue engineering is the fabrication of scaffolds that closely mimic the biomechanical properties of the tissues to be regenerated. Conventional fabrication techniques are not sufficiently suitable to control scaffold structure to modulate mechanical properties. Within novel scaffold fabrication processes 3D fiber deposition (3DF) showed a great potential for tissue engineering applications because of the precision in making reproducible 3D scaffolds, characterized by $100 \%$ interconnected pores with different shapes and sizes. Evidently, these features also affect mechanical properties. Therefore, in this study we considered the influence of different structures on dynamic mechanical properties of 3DF scaffolds. Pores were varied in size and shape, by changing fiber diameter, spacing and orientation, and layer thickness. With increasing porosity, dynamic mechanical analysis (DMA) revealed a decrease in elastic properties, such as dynamic stiffness and equilibrium modulus, and an increase of the viscous parameters, such as damping factor and creep unrecovered strain. Furthermore, the Poisson ratio was measured, and the shear modulus computed from it. Scaffolds showed an adaptable degree of compressibility between sponges and incompressible materials. As comparison, bovine cartilage was tested and its properties fell in the fabricated scaffold range. This investigation showed that viscoelastic properties of $3 \mathrm{DF}$ scaffolds could be modulated to accomplish mechanical requirements for tailored-tissue engineered applications (Moroni et al. 2006).

It has already been shown that the use of collagen-based scaffolds seeded with mesenchymal stem cells is a viable route to tissue engineering of hyaline cartilage using both in vitro and in vivo procedures. In the in vivo procedures, the scaffold has to provide both mechanical and biological support for the stem cells to grow and differentiate properly. The porous structure of the scaffold required for cell ingrowth brings about a major biomechanical complication, especially in the case of treating lesions on the load-bearing surfaces of large joints. Mechanical response of a porous scaffold is determined by the overall porosity, size and connectivity of the pores and the properties of the material forming the walls between pores. Since the last decade, tissue engineering has shown a sensational promise in providing more viable alternatives to surgical procedures for harvested tissues, implants and prostheses. Due to the fast development on biomaterial technologies, it is now possible for doctors to use patients' cells to repair orthopedic defects, such as focal articular cartilage lesions. In order to support the three-dimensional tissue formation, scaffolds made from biocompatible and bioresorbable polymers and composite materials, for providing temporary support of damaged body and cell structures have been developed recently. Although ceramic and metallic materials have been widely accepted for the development of implants, its non-resorbability and necessity of second surgical operation, which induces extra stress for the patients, limit their wide applications (Cheung et al. 2007).

Scaffolds used as wound dressings for tissue engineering are usually required to either dissolve or to be reabsorbed into the body after successful tissue regeneration. The scaffold should be biocompatible, biodegradable, highly porous with a large surface/volume ratio, mechanically strong, and processable into desired shapes (Chen et al. 2000). In addition, the threedimensional construct composed of scaffold/tissue should maintain sufficient mechanical strength during the in vitro and in vivo growth and remodeling process. Many methods to prepare porous three-dimensional biodegradable scaffolds have been developed in tissue engineering, including gas forming, fiber extrusion and bonding, three-dimensional printing, phase separation, emulsion freeze-drying and porogen leaching (Chen et al. 2002a). Recently, a new kind of hybridization technique was developed and used to fabricate porous hybrid scaffolds by combining synthetic and natural biodegradable polymers, such as poly(D,L-lactic-coglycolic acid) (PLGA) and collagen (Chen et al. 2000). In the hybrid scaffold, the synthetic biodegradable polymers are easily formed into desired shape with good mechanical strength, and the duration of degradation can be estimated. Despite these advantages, the scaffolds derived from synthetic polymers are insufficient for cellrecognition signals and their hydrophobic properties obstruct smooth cell seeding. In contrast, naturally derived polymers have the potential advantages of specific cell interactions and a hydrophilic nature, but possess poor mechanical properties. Thus, these two kinds of biodegradable polymers have been hybridized to combine the advantageous properties of both constituents. Furthermore, hybridization with collagen facilitates cell seeding and spatial cell distribution, and promotes cell immigration and neo-angiogenesis (Chen et al. 2002b).

In this paper, the effect of modification of the cross-linked collagen I with hyaluronic acid, nanometersize hydroxyapatite particles and chitosan nanofibers on the mechanical response of the lyophilized porous scaffold construct has been investigated in the dry state at 


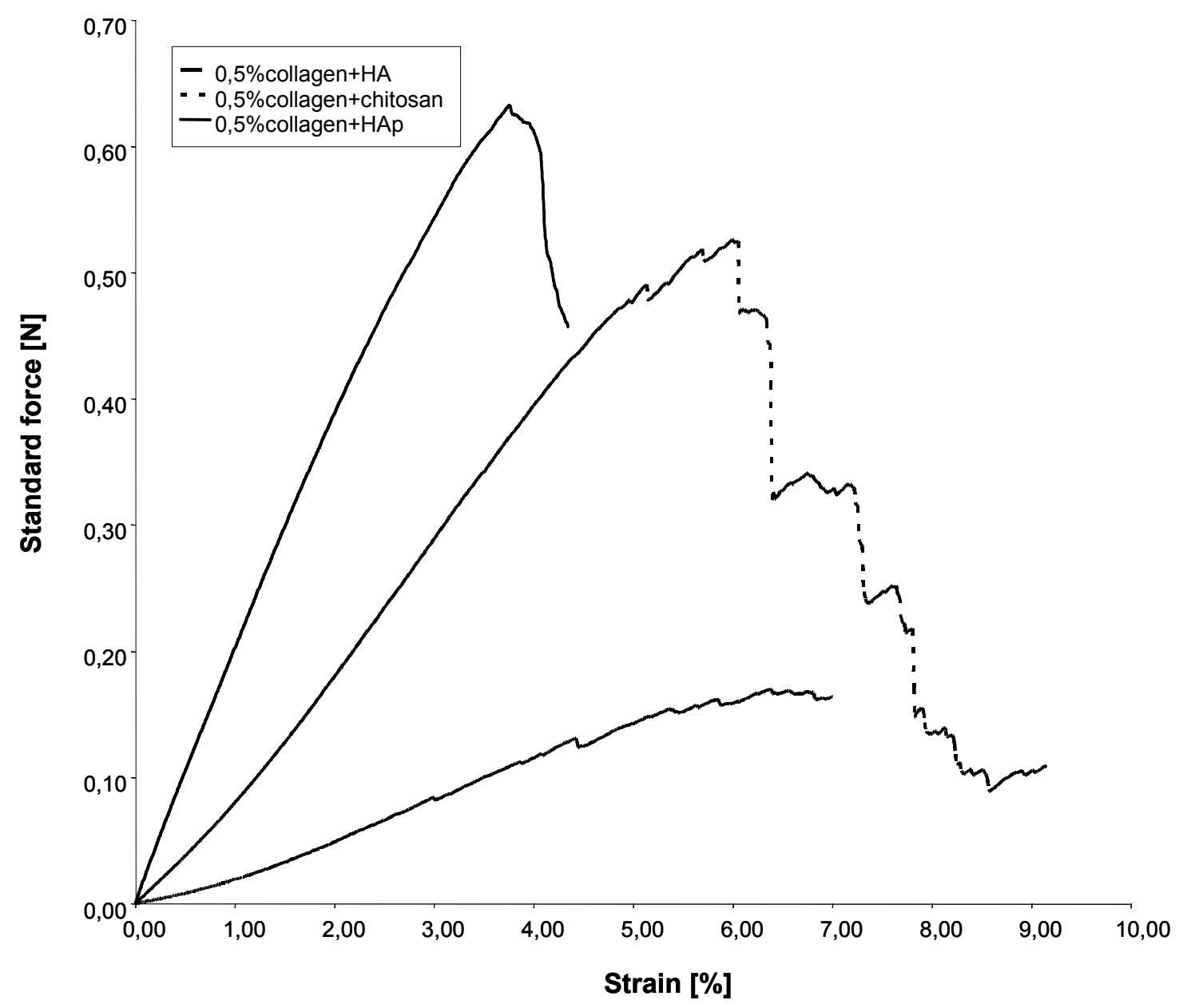

Fig. 1. Deformation curves of the modified cross-linked collagen.

\section{Methods}

Mechanical measurements were performed using Zwick Z010 (Zwick Roel, Germany) tensile tester employing $100 \mathrm{~N}$ measuring cell under tensile loading in a temperature chamber at $37{ }^{\circ} \mathrm{C}$ and $70 \% \mathrm{RH}$ at the cross-head speed of $50 \mathrm{~mm} / \mathrm{min}$. Five specimens for each composition and type of loading were used. Rectangular bars were cut out of the flat porous scaffold sheets and loaded in tension until fracture. The presented values are the average values calculated from 5 measurements. Standard deviation of $10 \%$ was obtained for all the measurements. ANOVA analysis $(\mathrm{p}<<0.05)$ was performed to determine whether the addition of the various agents causes statistically significant differences between the scaffold materials using the Origin 7.0 software (OriginLab, USA). Morphology of the individual porous scaffolds has been observed using SEM (Phillips, Quanta 600, FEI, Czech Republic) and Laser Confocal Microscope (Olympus LCM 3000, Olympus, pores. The appearance of the different modified collagen foams is shown in Fig. 2. The pore walls fractured apparently in a brittle manner. Added hydroxyapatite nanoparticles appear to be dispersed uniformly in the collagen matrix (Fig. 3). Similarly to hyaluronic-acidmodified collagen, fibril-like objects were observed near the fracture surface containing also dispersed hydroxyapatite particles. The foam structure similar to neat collagen has been observed for the hyaluronic acid modified cross-linked collagen I (Fig. 4), except for some fiber-like objects occurring on the fracture surface. SEM of the collagen modified with chitosan nanofibers revealed the interesting structure of this nanocomposite (Fig. 5). Apparently, the chitosan nanofibers formed a loosely connected 3D fiber network contained within the collagen pore walls. Qualitatively, the adhesion between the chitosan nanofibers and collagen matrix was very good, resulting in fibers bridging the fractured collagen walls. 

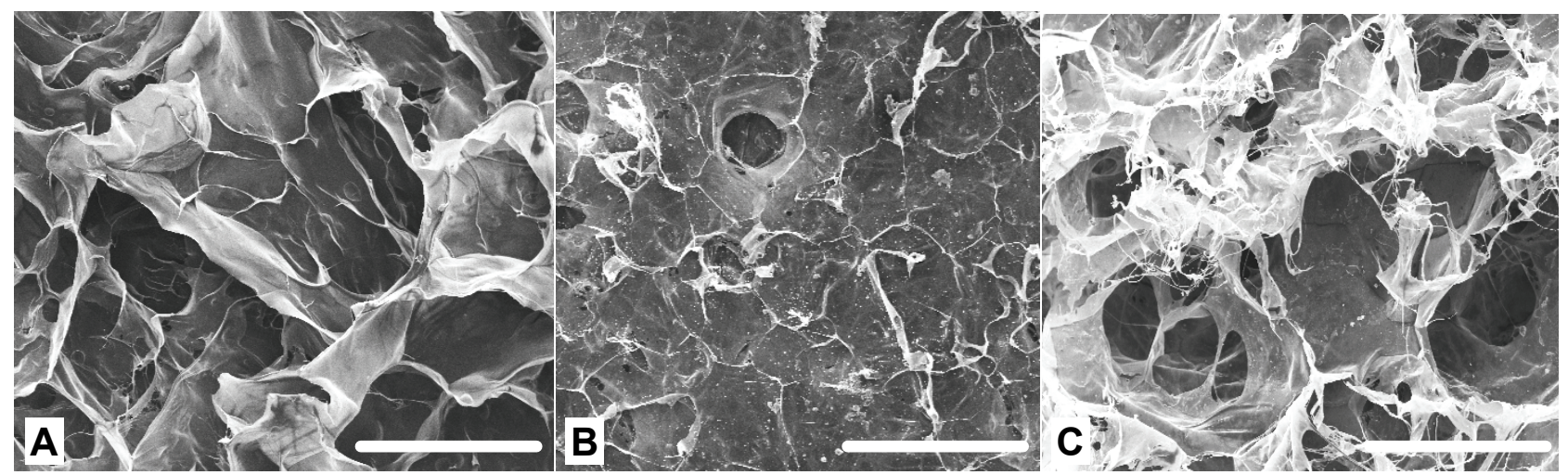

Fig. 2. SEM micrographs of various porous scaffold structures. (A) collagen/chitosan nanofiber scaffold, (B) collagen/hydroxyapatite scaffold, (C) collagen/hyaluronic acid scaffold. Lyophilized specimens were gold-coated prior to observation. Bars in the right-hand lower corner represent $100 \mathrm{~nm}$.
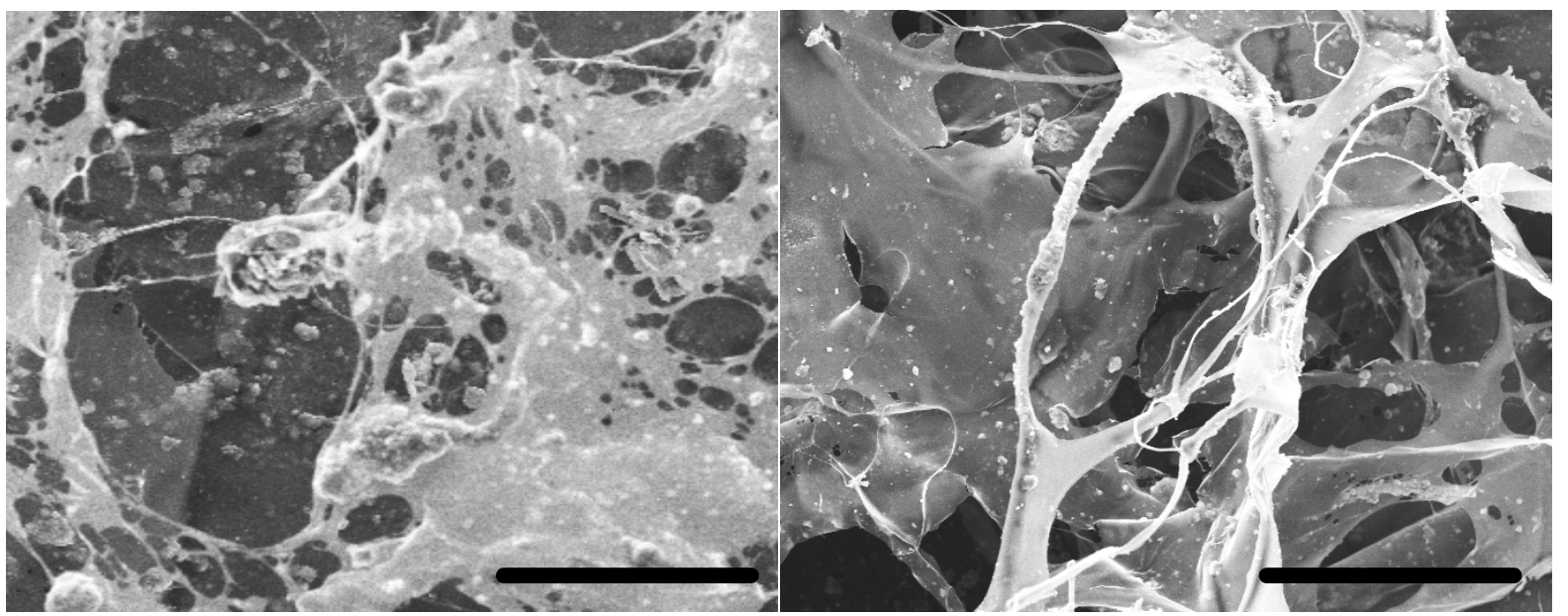

Fig. 3. Uncross-linked and cross-linked collagen reinforced with hydroxyapatite nanoparticles. Bar in the right-hand lower corner represents $200 \mathrm{~nm}$.

Table 1. Tensile elastic modulus of hyaline cartilage tissue and various porous scaffolds.

\begin{tabular}{|c|c|}
\hline Tissue material & $\mathrm{E}_{\mathrm{t}}[\mathrm{GPa}]$ \\
\hline Human femoral condyle cartilage* & 0,5 \\
\hline Bovine articular cartilage* & 0,9 \\
\hline \multicolumn{2}{|l|}{ Scaffold material } \\
\hline$X$-Linked collagen $I * *$ & $20 \pm 2$ \\
\hline $\begin{array}{l}X \text {-Linked collagen I }+ \text { hydroxyapatite } \\
\text { nanoparticles } * *\end{array}$ & $200 \pm 14$ \\
\hline$X$-Linked collagen $I+H A * *$ & $18 \pm 2$ \\
\hline $\begin{array}{l}X \text {-Linked collagen } I+\text { chitosan } \\
\text { nanofibers } * *\end{array}$ & $85 \pm 6$ \\
\hline
\end{tabular}

* aggregate modulus measured at low strain rates (Martin et al. 1998)

** lyophilizate with $75 \%$ porosity, measured at $37^{\circ} \mathrm{C}, 70 \%$ relative humidity, cross-head speed $50 \mathrm{~mm} / \mathrm{min}$
In agreement with the simple composite model of mechanical response of articular cartilage (Schwartz et al. 1994), the stiffer the modifying agent the steeper the build-up of stress with increasing extension of the modified cross-linked collagen I (Table 1), i.e., the greater the elastic modulus. Since all the materials investigated exhibited a similar porosity as well as similar size of the pores, most probably the observed increase of the foam rigidity can be attributed to the enhancement of the elastic modulus of the material forming the pore walls.

In all cases, the adhesion between the components seemed very good and no dewetting of the secondary component has been observed for the hydroxyapatite nanoparticle and chitosan-nanofibermodified collagen at the ultimate deformation. In the case of hyaluronic acid (HA)-modified collagen, homogeneous structure of the pore walls was observed similarly to neat collagen, suggesting good mixing between the two 

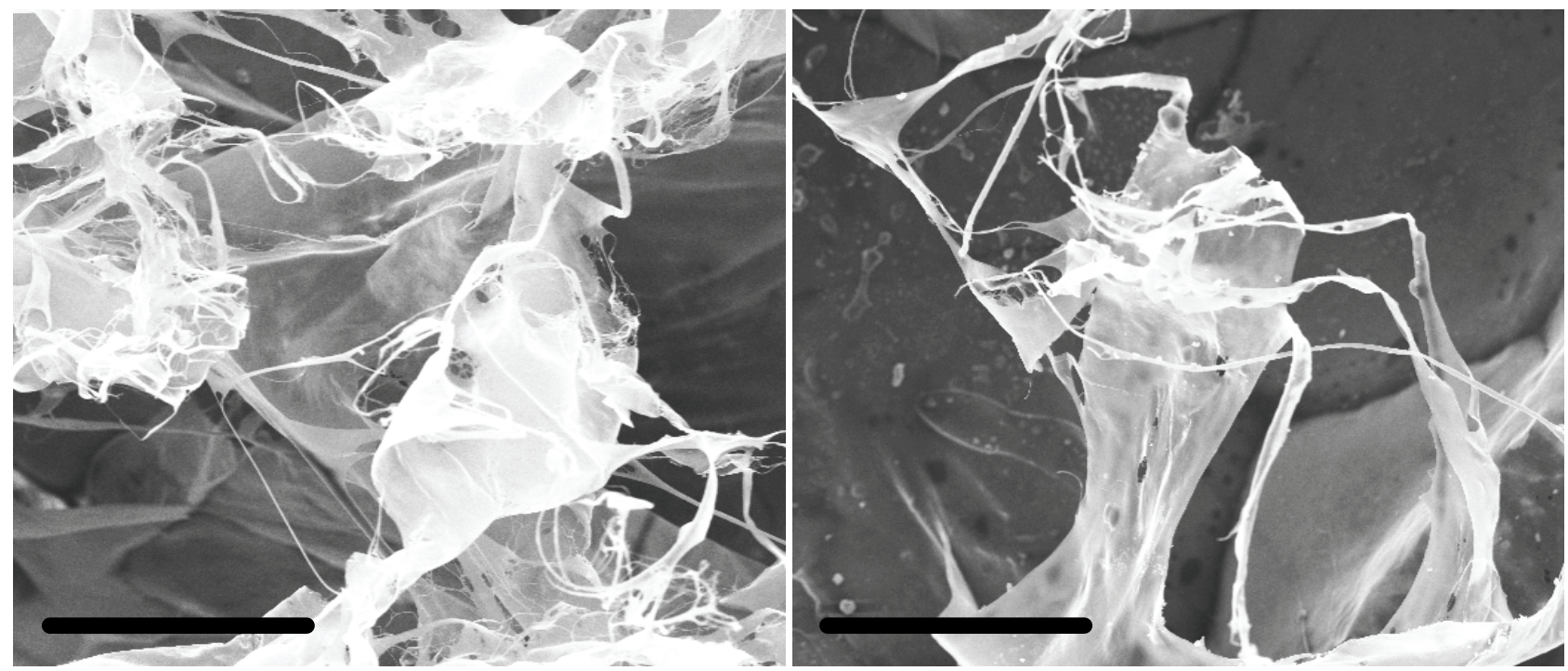

Fig. 4. Uncross-linked and cross-linked collagen modified with hyaluronic acid. Bar in the left-hand lower corner represents $200 \mathrm{~nm}$.

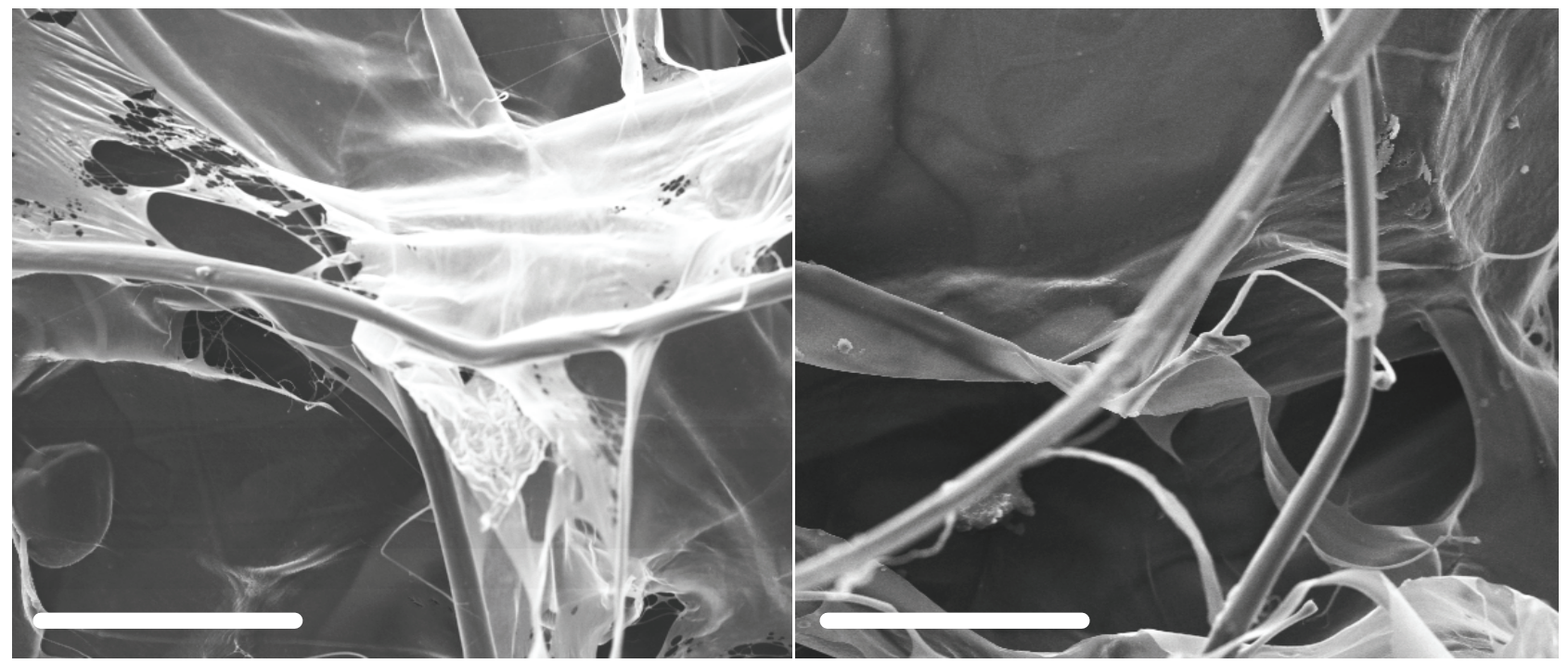

Fig. 5. Uncross-linked and cross-linked collagen reinforced with chitosan nanofibers. Bar in the left-hand lower corner represents 200 $\mathrm{nm}$.

polymers. In this case, one can consider 3 possible molecular structures of these blends. In the first case, HA was assumed to be attached to the collagen network during the cross-linking reaction forming bulky pendant side chains. This disturbed the regularity of the cross-linked collagen network and caused reduction of the elastic modulus compared to neat collagen due to increased number of elastically ineffective chains as well as increase in the ultimate deformation. In the second case, collagen and HA were assumed to form two independent interpenetrating networks on the molecular level. In the third case, HA was assumed to be dispersed in the collagen matrix without any chemical bond to the collagen acting as a poor plasticizer for the collagen network.

\section{Conclusions}

In this paper, the effect of modification of the cross-linked collagen I with hyaluronic acid, hydroxyapatite nanoparticles and chitosan nanofibers on the mechanical response of the compact scaffold material and the lyophilized porous scaffold construct has been investigated in the dry state at $37{ }^{\circ} \mathrm{C}$ under tensile loading. The results showed that the addition of hyaluronic acid reduced the tensile elastic modulus and slightly increased the ultimate deformation of crosslinked collagen I under the test conditions used. On the other hand, addition of hydroxyapatite nanoparticles and chitosan nanofibers, respectively, increased the elastic 
modulus of the modified collagen ten-fold and four-fold, respectively. The ultimate tensile deformation has been decreased by hydroxyapatite and increased by the chitosan nanofibers. It can be concluded that enhancing the stiffness of the compact scaffold material by adding rigid chitosan nanofibers can improve the resistance of the porous scaffolds against mechanical loading. SEM micrographs revealed the effect of the respective additives on the pore structure of the lyophilized collagen foams. Collagen modified with chitosan nanofibers exhibited the most uniform pore structure. Moreover, chitosan nanofibers seemed to enhance mesenchymal cell growth compared to neat collagen I.

\section{Acknowledgements}

Financial support of this research under NPV II project 2B06130 and project MS 0021630501 from the Ministry of Education, Youth and Sports is greatly appreciated. This work was also supported by Grant Agency AGELMH. Part of this research has been supported by the industrial partners from the Laboratory of Biomaterials at the Institute of Materials Chemistry.

\section{References}

CHEN AC, BAE WC, SCHINAGL RM, SAH RL: Depth- and strain-dependent mechanical and electromechanical properties of full-thickness bovine articular cartilage in confined compression. J Biomech 34: 1-12, 2001.

CHEN G, AKAHANE D, KAWAZOE N, YAMAMOTO K, TATEISHI T: Chondrogenic differentiation of mesenchymal stem cells in a leakproof collagen sponge. Mater Science Engin 45: 389-398, 2007.

CHEN G, USHIDA T, TATEISHI T: A biodegradable hybrid sponge nested with collagen microsponges. $J$ Biomed Mater Res 51: 273-279, 2000.

CHEN G, USHIDA T, TATEISHI T: Scaffold design for tissue engineering. Macromol Biosci 2: 67-77, 2002a.

CHEN G, USHIDA T, TATEISHI T: Biodegradable porous scaffolds for tissue engineering. J Artif Organs 5: 77-83, 2002 b.

CHEUNG HY, LAU KT, LU TP, HUI D: A critical review on polymer-based bio-engineered materials for scaffold development. Composites Part B: Engineering 38: 291-300, 2007.

COURTNEY T, SACKS MS, STANKUS J, GUAN J, WAGNER WR: Design and analysis of tissue ingineering scaffolds that mimic soft tissu mechanical anisotropy. Biomaterials 27: 3631-3638, 2006.

DOROTKA R, WINDBERGER U, MACFELDA K, BINDREITER U, TOMA, NEHRER S: Repair of articular cartilage defects treated by microfracture and a three-dimensional collagen matrix. Biomaterials 26: $3617-$ $3629,2005$.

FANG Z, STARLY B, SUN W: Computer-aided characterization for effective mechanical properties of porous tissue scaffolds. Computer-Aided Design 37: 65-72, 2005.

KORHONEN RK, LAASANEN MS, TOYRAS J, RIEPPO J, HIRVONEN J, HELMINEN HJ, JURVELIN JS: Comparison of the equilibrium response of articular cartilage in unconfined compression, confined compression and indentation. J Biomech 35: 903-909, 2002.

LEE JH, PARK TG, PARK HS, LEE DS, LEE YK, YOON SC, DO NAM J: Thermal and mechanical characteristics of poly(L-lactic acid)-nanocomposite scaffold. Biomaterials 24: 2773-2778, 2003.

LI LP, BUSCHMANN MD, SHIRAZI-ADL A: A fibril reinforced nonhomogeneous poroelastic model for articular cartilage: inhomogeneous response in unconfined compression. J Biomech 33: 1533-1541, 2000.

LEE SB, KIM YH, CHONG MS, LEE YM: Preparation and characteristics of hybrid scaffolds composed of $\beta$-chitin and collagen. Biomaterials 25: 2309-2317, 2004.

MARTIN RB, BURR DB, SHARKEY NA: In: Skeletal Tissue Mechanics, Springer, New York, 1998, pp. $278-289$.

MORONI L, DE WIJN LR, VAN BLITTERSWIJK CA: 3D fiber-deposited scaffolds for tissue engineering: Influence of pores geometry and architecture on dynamic mechanical properties. Biomaterials 27: 974-985, 2006.

MOW VC, ZHU W, RATCLIFFE A: Structure and function of articular cartilage. In: Basic Orthopaedic Biomechanics, Raven Press, New York, 1991, pp 143-198.

RISBUD MV, SITTINGER M: In vitro expression of cartilage-specific markers by chondrocytes on a biocompatible hydrogel: implications for engineering cartilage tissue. Cell Transplant. 10: 755-763, 2001. 
RISBUD MV, SITTINGER M: Tissue engineering: advances in in- vitro cartilage generation. Trends Biotechnol 20: 351-355, 2002.

SCHWARTZ MH, LEO PH, LEWIS JL: A microstructural model for the elastic response of articular cartilage. $J$ Biomech 27: 865-873, 1994.

SIMHA NK, FEDEWA M, LEO PH, LEWIS JH, OEGEMA T: A composites theory predicts the dependence of stilness of cartilage culture tissues on collagen volume fraction. J Biomech 32: 503-509, 1999.

TEMENO JS, MIKOS AG: Injectable biodegradable materials for orthopedic tissue engineering. Biomaterials 21: 2405-2412, 2000.

TOYRAS J, LYYRA-LAITINEN T, NIINIMAKI M, LINDGREN R, NIEMINEN MT, KIVIRANTA I, JURVELIN JS: Estimation of the Young's modulus of articular cartilage using an arthroscopic indentation instrument and ultrasonic measurement of tissue thickness. J Biomech 34: 251-256, 2001.

WILLIAMS RJ, DREESE JC, CHEN CT: Chondrocyte survival and material properties of hypothermically stored cartilage. Am J Sports Med 32: 132-139, 2004.

\section{Corresponding author}

J. Jančár̆, Institute of Materials Chemistry, University of Technology, Purkyňova 118, 61200 Brno, Czech Republic. Email: jancar@fch.vutbr.cz 